\title{
Snakebites in Suriname: Evaluation of the Protocolled Administration of Anti-Snake Venom in a Tertiary Care Setting
}

\author{
Navin Ramdhani, ${ }^{1 \star}$ Simone Jonker, ${ }^{1}$ Kevin van 't Kruys, ${ }^{2}$ Rakesh Bansie, ${ }^{3}$ and Wilco Zijlmans ${ }^{4,5}$ \\ ${ }^{1}$ Department of Intensive Care Unit, Academic Hospital Paramaribo, Paramaribo, Suriname; ${ }^{2}$ Academic Pediatric Center Suriname, Academic \\ Hospital Paramaribo, Paramaribo, Suriname; ${ }^{3}$ Department of Internal Medicine, Academic Hospital Paramaribo, Paramaribo, Suriname; ${ }^{4}$ Scientific \\ Research Center Suriname, Academic Hospital Paramaribo, Paramaribo, Suriname; ${ }^{5}$ Faculty of Medical Sciences, Anton de Kom University of \\ Suriname, Paramaribo, Suriname
}

\begin{abstract}
Venomous snakebites regularly occur in Suriname, a middle-income country located on the north coast of South America. Officially reported data on incidence and mortality are lacking. The aim of this retrospective study was to assess whether the use of our national snakebite protocol with selective administration of anti-snake venom (ASV) in patients with signs of snakebite envenoming improved clinical outcome as measured by mortality and length of stay (LOS) in the hospital. Medical records of all patients admitted at the Academic Hospital Paramaribo from 2013 to 2015, before and after the introduction of the protocol, with signs of snakebite envenoming, were reviewed for demographics, snakebite characteristics, mortality, length of hospital stay, administration of ASV, and occurrence of complications. Secondary outcome measures were the development of late complications due to a snakebite. Sixty-eight and 76 patients in 2013 and 2015, respectively, with venomous or potentially venomous snakebites were identified. One patient (1.5\%) in 2013 and 29 patients (38.2\%) in 2015 received ASV. In 2013 one patient died: deterioration of renal function occurred before protocolled ASV administration. No deaths were reported in 2015 . There was no difference in the overall length of hospital stay between 2013 and 2015 or in the total number of late complications. In 2015, the mean LOS ( \pm SD) for patients who did not receive ASV $(n=47)$ was significantly lower than that for patients who received ASV $(n=29), 2.15 \pm$ 2.27 versus $5.31 \pm 5.53$ days, respectively $(P=0.001)$. The mean LOS $( \pm$ SD) for patients who did not receive ASV in 2013 $(n=67)$ and $2015(n=47)$ was $4.06 \pm 5.44$ and $2.15 \pm 2.27$ days, respectively, which also differed significantly $(P=0.025)$. The protocolled evaluation of snakebite victims resulted in more patients being admitted to the intensive care unit and receiving ASV and a shorter length of hospital stay for the patients who did not receive ASV, and no difference in the occurrence of complications was observed in Suriname's largest hospital responsible for the acute care of snakebite victims.
\end{abstract}

\section{INTRODUCTION}

Snakebite envenoming is a major cause of injury worldwide, with an estimated 1.8-2.7 million cases and 81,000-138,000 deaths annually. Higher incidence of snakebite envenoming and increasing mortality and morbidity rates are found in tropical and subtropical areas such as South and Southeast Asia, sub-Saharan Africa, and Latin America. The economic burden is considerable, as victims mostly live in poor and rural communities. ${ }^{1}$ Venomous snakebites regularly occur in Suriname, a middle-income country located on the north coast of South America and largely covered by vast tropical rainforest. However, officially reported data on incidence or mortality are lacking. In the northern tropical rainforest region of South America, venomous snakes belonging to the genus Bothrops, Crotalus, Lachesis, and Micrurus are medically most relevant. ${ }^{2}$ In Suriname, nine toxic snake species are found, the two most common ones being Bothrops atrox and Lachesis muta, both belonging to the Viperidae family of snakes (http:// apps.who.int/bloodproducts/snakeantivenoms/database/). Venom of these snakes can induce serious local and systemic complications, of which venom-induced consumption coagulopathy (VICC) is feared because of the procoagulant toxin-induced consumption of clotting factors that may result in life-threatening hemorrhage and death. ${ }^{1,3,4}$ Anti-snake venom (ASV) is the recommended treatment for snake envenoming, but its administration is not without risk because of potential severe adverse effects. One of the major indications

*Address correspondence to Navin Ramdhani, Department of Intensive Care Unit, Academic Hospital Paramaribo, Flustraat volgt, Paramaribo, Suriname. E-mail: nramdhani@azp.sr for treatment with ASV is development of significant VICC. ${ }^{5}$ Colleagues from our neighboring country French Guiana recently published on local predictors of complications of snakebite envenomation. ${ }^{6}$ Because of the high snakebite mortality rate, they have argued for a re-evaluation of their snakebite management protocols and the use of ASV. ${ }^{7}$

Since 2014, the Academic Hospital Paramaribo (AHP) in Suriname has implemented a national protocol partly based on WHO guidelines for the treatment of snakebites (see Appendix 1 for protocol summary), resulting in a systematic approach to snakebites and the standardized administration of ASV in selected patients.

There are, however, no randomized controlled trials studying the efficacy of ASV in the treatment of VICC in comparison to a placebo. ${ }^{4,8,9}$ At this time, conducting a placebocontrolled trial to show the efficacy of ASV is unethical, as the WHO stated in 2014 that there are enough welldocumented data on the beneficial effect of ASV in South America. Therefore, it is recommended by the WHO and unacceptable to withhold ASV from a patient with signs of serious envenomation.

This retrospective observational study aimed to assess whether the use of our protocol with selective administration of ASV in patients with signs of snakebite envenoming improved patient assessment, patient care, and clinical outcome as measured by mortality and length of stay (LOS) in the hospital. The secondary outcome measures were the development of late complications, defined as signs that were directly related to the snakebite but were not present on admission and the occurrence of adverse effects following ASV administration. 


\section{METHODS}

Setting. The AHP is the largest hospital in Suriname with the largest intensive care unit (ICU). This ICU is level 3 equipped and facilitates treatment of patients post-cardiothoracic surgery or following neurosurgery, and those with severe sepsis or severe trauma and has over a 1,000 admissions every year. The AHP is responsible for the acute care of more than $70 \%$ of all health-care seeking snakebite victims in the country. The ICU is hereby a crucial link in the integrated care of these patients and is responsible for respiratory and hemodynamic stabilization, patient monitoring, and administration of ASV. At the AHP, a polyvalent ASV is used, made with the venoms of B. atrox, L. muta, Bothrops asper, and Crotalus durissus (Suero Antiofidico Polivalente Liofilizado, Probiol, Bogotá, Columbia).

Data collection and analysis. Medical record data from years 2013 and 2015, 1 year before and 1 year after the introduction of the protocol for the treatment of snakebites in 2014 , of all patients with a snakebite admitted at the AHP were assessed. The year 2014 was excluded to reduce the implementation bias which could be present upon the introduction of the protocol for treatment of snakebites. The Institutional Review Board of the AHP gave ethical approval for this study.

Information was collected from medical records on patient demographics (gender, age, and comorbidity) and snakebite characteristics, including species, number of bites, location, and complications after the snakebite. A venomous snakebite was defined as one with a positive identification of a venomous snake or a snakebite without the positive identification of a venomous snake but with the clinical and/or laboratory signs of snakebite envenoming.

Furthermore, data were collected on the length of hospital stay, mortality, and occurrence of complications. Complications consisted of local and systemic complications. Local complications include blistering, edema, cellulitis, abscess formation, necrosis, and compartment syndrome. Systemic complications consisted of hypotension, rhabdomyolysis (as determined via serum creatine kinase measurements), acute kidney injury (AKI) (as determined by a rise in serum creatinine above baseline or above previously known value in case of existing chronic renal failure), and VICC, among others. Laboratory data were collected for metabolic and coagulation abnormalities (prothrombin time/international normalized ratio (INR) and activated partial thromboplastin time). Data on the indications for ASV were collected for 2015. The administered dose for ASV depended on the species identified. Standard administration consisted of $20 \mathrm{~mL}$ ASV. In the presence of either repeated bites by Lachesis muta or severe neurotoxicity due to Micrurus species, $80 \mathrm{~mL}$ of ASV would be administered. The indications for ASV use, regardless of the snake species, due to systemic envenoming are hemostatic abnormalities (including VICC), neurotoxic signs, cardiovascular abnormalities, AKI, and rhabdomyolysis. The indications for ASV use, regardless of the snake species, due to local envenoming include swelling involving more than half of the involved limb, progressive swelling, and development of enlarged and tender lymph nodes draining the affected limb. The timing and occurrence of adverse effects caused by ASV administration was also recorded. Patient data were further assessed on whether fresh frozen plasma (FFP) and/or vitamin $\mathrm{K}$ was administered.
Mortality and length of hospital stay, as primary outcome measures, were defined as the absolute number of fatal cases and days of hospitalization (mean $\pm S D$ ), respectively. A patient discharged within 24 hours would have a 1-day LOS.

Statistical analysis. All the raw data were entered on Excel 2013 spreadsheets (Microsoft Corporation, Redmond, WA). IBM SPSS Statistics for Windows (version 21; IBM Corp., Armonk, NY) was used for statistical analysis. Descriptive analyses were performed. Chi-square test or Fisher's exact test was used for categorical variables, and student's $t$-test was used for continuous variables. A $P$-value $<0.05$ was considered statistically significant.

\section{RESULTS}

Table 1 presents the characteristics of 144 cases of victims of poisonous or potentially poisonous snakebites presenting at the emergency department of the AHP: 68 in 2013 and 76 in 2015. Two patients in 2015 were bitten by a Boa constrictor and Eunectes murinus, respectively, and were excluded from analysis. In about half the cases in both years, patients could not identify the snake responsible for the bite. In cases where the snake was recognized during the 2 years, $B$. atrox was involved in $33.8 \%$ and $39.5 \%$ of cases in 2013 and 2015 , respectively. Lachesis muta was involved in $8.8 \%$ and $6.6 \%$ of cases for the years 2013 and 2015 respectively. Bothrops atrox and $L$. muta were identified in $48.3 \%$ and $6.9 \%$ of patients, respectively, who received ASV in 2015. For the patients who did not receive ASV in 2015, B. atrox and L. muta were identified in $6.4 \%$ and $34 \%$ of cases, respectively. All admitted patients of both years presented with one bite in one of their extremities, most often in the lower extremities. The clinical presentation at admission comprised mostly local manifestations. Infectious complications were the second most frequently encountered complication. There were no significant differences between the patients in 2013 and 2015, apart from hypertension which was more frequently present in patients from 2015.

VVIC and ASV administration. Table 2 presents the laboratory results and interventions: in 2015 , significantly more patients with known coagulation studies at admission had prolonged INR $\geq 1.6(P=0.047)$ and more patients received ASV $(P<0.001)$, than in 2013 . Of the 29 patients in whom ASV was administered in 2015, 26 (89.7\%) received it within 24 hours after presentation at the emergency room. Only one patient in 2015 received a repeated dose of ASV because of prolonged coagulation times. Adverse events during ASV administration were noted in 13 patients: in 2013, the one patient who received ASV developed itching and tachycardia; in 2015 , three patients developed urticaria, itching was reported in only one patient, and fever and chills were reported in six patients. All patients received proper medication according to protocol. The administration of ASV to these patients was not cancelled because of the adverse effects. There was no significant difference between 2013 and 2015 in the number of patients who received FFP and vitamin $\mathrm{K}$ for coagulation disorders.

The patients who received ASV in 2015 had statistically more hematologic and metabolic complications than the patients who did not receive ASV (Table 3). In 2015, one pediatric patient received ASV in the general pediatric ward because of VICC as there was no available ICU bed at that moment. 
TABLE 1

Characteristics of snakebite victims presented at the emergency department of the Academic Hospital Paramaribo from 2013 to 2015

\begin{tabular}{|c|c|c|c|}
\hline Total cases $(n=144)$ & $2013(N=68)$ & $2015(N=76)$ & $P$-value \\
\hline Male, $n(\%)$ & $43(63.2)$ & 49 (64.5) & $0.548^{*}$ \\
\hline Age (years), mean $\pm S D$ & $30.81 \pm 19.51$ & $35.29 \pm 19.13$ & $0.167 \dagger$ \\
\hline \multicolumn{4}{|l|}{ Comorbidities, $n(\%)$} \\
\hline Diabetes mellitus & $3(4.4)$ & $6(7.9)$ & $0.389^{\star}$ \\
\hline Hypertension & $5(7.3)$ & $15(19.8)$ & $0.032^{*}$ \\
\hline $\begin{array}{l}\text { Asthma/chronic obstructive pulmonary } \\
\text { disease }\end{array}$ & $1(1.5)$ & $0(0)$ & $0.289^{*}$ \\
\hline Other & $1(1.5)$ & $4(5.3)$ & $0.215^{\star}$ \\
\hline No comorbidities, $n$ (\%) & 37 (54.4) & 48 (63.2) & $0.287^{*}$ \\
\hline Unknown comorbidities, $n$ (\%) & $24(35.3)$ & $8(10.5)$ & $0.000^{*}$ \\
\hline \multicolumn{4}{|c|}{ Identification of poisonous snakes $(n=144), n(\%)$} \\
\hline Identified poisonous snake & $30(44.1)$ & $37(48.7)$ & 0.352 \\
\hline $\begin{array}{l}\text { Not identified/presumably poisonous } \\
\text { snake }\end{array}$ & $38(55.9)$ & $39(51.3)$ & \\
\hline Bothrops atrox & $23(33.8)$ & $30(39.5)$ & 0.811 \\
\hline Bothrops bilineatus & $1(1.5)$ & $1(1.3)$ & \\
\hline Crotalus durissus & $0(0)$ & $1(1.3)$ & \\
\hline Lachesis muta & $6(8.8)$ & $5(6.6)$ & \\
\hline Number of bites/person & 1 & 1 & \\
\hline \multicolumn{4}{|l|}{ Localization of snakebites $(n=144), n(\%)$} \\
\hline Hand & $14(20.6)$ & $14(18.4)$ & $0.894^{\star}$ \\
\hline Feet & 45 (66.2) & 53 (69.7) & \\
\hline Upper leg & $8(11.8)$ & $7(9.2)$ & \\
\hline Lower leg & $1(1.5)$ & $2(2.6)$ & \\
\hline \multicolumn{4}{|c|}{ Time to hospital (hours) $(n=130$, missing $=14), n(\%)$} \\
\hline$\leq 6$ & $42(72.4)$ & 55 (76.4) & $0.406^{*}$ \\
\hline $6>$ hours $\leq 24$ & $9(15.5)$ & $13(18.1)$ & \\
\hline$>24$ & $7(12.1)$ & $4(5.6)$ & \\
\hline \multicolumn{4}{|l|}{ Signs and symptoms of envenoming, $n(\%)$} \\
\hline Local symptoms & 64 (94.1) & 71 (93.4) & $0.486^{*}$ \\
\hline Hematologic & $8(11.8)$ & $12(15.8)$ & $0.326^{\star}$ \\
\hline Neurological & $0(0)$ & $2(2.6)$ & $0.178^{\star}$ \\
\hline Hemodynamic & $0(0)$ & $1(1.3)$ & $0.343^{\star}$ \\
\hline Metabolic & $1(1.5)$ & $5(6.6)$ & $0.126^{\star}$ \\
\hline Infectious & $14(20.6)$ & $15(19.7)$ & $0.899^{\star}$ \\
\hline Gastrointestinal & $1(1.5)$ & $1(1.3)$ & $0.937^{*}$ \\
\hline Other & $0(0)$ & $1(1.3)$ & $0.343^{*}$ \\
\hline No symptoms & $1(1.5)$ & 3 (3.9) & $0.367^{*}$ \\
\hline Missing & $3(4.4)$ & $0(0)$ & $0.064^{*}$ \\
\hline
\end{tabular}

Mortality. One patient admitted for a venomous snakebite died in 2013. The patient was a 43-year-old woman who presented within 2 hours of a possible $B$. atrox bite to the emergency department with pain and swelling of the left foot. She left against medical advice before any treatment could be given to visit a traditional healer. She returned the day after with progressive pain and swelling and multi-organ failure: acute kidney failure, elevated liver enzymes, severe coagulopathy, and metabolic dysregulation. She was started on dialysis and received packed red blood cells and FFP. The

TABLE 2

Laboratory results and interventions in 144 snakebite victims presented at the emergency department of the Academic Hospital Paramaribo from 2013 to 2015

\begin{tabular}{|c|c|c|c|}
\hline Total cases $(N=144)$ & $2013(n=68), n(\%)$ & $2015(n=76), n(\%)$ & $P$-value \\
\hline APTT > 48 seconds & $13(19.1)$ & 25 (32.9) & $0.096^{\star}$ \\
\hline $\mathrm{APTT} \leq 48$ seconds & 47 (69.1) & 47 (61.8) & \\
\hline APTT unknown & $8(11.8)$ & $4(5.3)$ & \\
\hline INR $\geq 1.6$ & $17(25)$ & $30(39.5)$ & $0.047^{*}$ \\
\hline $\mathrm{INR}<1.6$ & 39 (57.4) & $41(53.9)$ & \\
\hline INR unknown & $12(17.6)$ & $5(6.6)$ & \\
\hline ASV administration & $1(1.5)$ & 29 (38.2) & $<0.001^{\star}$ \\
\hline$<24$ hours until ASV administration & $0(0)$ & $26(34.2)$ & $0.133^{*}$ \\
\hline$\geq 24$ hours until antivenom administration & $1(1.5)$ & $3(3.9)$ & \\
\hline $\begin{array}{l}\text { Adverse events during antivenom } \\
\text { administration }\end{array}$ & $1(1.5)$ & $13(17.1)$ & - \\
\hline Fresh frozen plasma administration & $51(75)$ & 64 (84.2) & $0.169^{*}$ \\
\hline Vitamin $\mathrm{K}$ administration & $9(13.2)$ & $15(19.7)$ & $0.296^{*}$ \\
\hline
\end{tabular}

APTT = activated partial thromboplastin time; ASV = anti-snake venom; INR = international normalized ratio. ${ }^{\star}$ Chi-square test. 
TABLE 3

Characteristics of patients in 2015

\begin{tabular}{|c|c|c|c|}
\hline \multirow[b]{2}{*}{2015} & \multicolumn{2}{|c|}{ Administration anti-snake venom } & \multirow[b]{2}{*}{$P$-value } \\
\hline & Yes $(n=29)$ & No $(n=47)$ & \\
\hline Male, $n(\%)$ & $20(69)$ & $29(61.7)$ & $0.52^{\star}$ \\
\hline Age (years), mean $\pm S D$ & $38.97 \pm 17$ & $33.02 \pm 20.18$ & $0.19 \dagger$ \\
\hline \multicolumn{4}{|l|}{ Identification of poisonous snakes, $n(\%)$} \\
\hline Identified poisonous snake & $17(58.6)$ & $20(42.6)$ & $0.173^{\star}$ \\
\hline $\begin{array}{l}\text { Not identified/presumably poisonous } \\
\text { snake }\end{array}$ & $12(41.4)$ & $27(57.4)$ & \\
\hline Bothrops atrox & $14(48.3)$ & $16(34)$ & \\
\hline Bothrops bilineatus & $1(3.4)$ & $0(0)$ & \\
\hline Crotalus durissus & $0(0)$ & $1(2.1)$ & \\
\hline Lachesis muta & $2(6.9)$ & $3(6.4)$ & \\
\hline \multicolumn{4}{|l|}{ Signs and symptoms of envenoming, $n(\%)$} \\
\hline Local symptoms & $29(100)$ & $42(89.4)$ & $0.069^{\star}$ \\
\hline Hematologic & $10(34.5)$ & $2(4.3)$ & $0.00^{\star}$ \\
\hline Neurological & $0(0)$ & $2(4.3)$ & $0.260^{\star}$ \\
\hline Hemodynamic & $0(0)$ & $1(2.1)$ & $0.429^{\star}$ \\
\hline Metabolic & $5(17.2)$ & $0(0)$ & $0.003^{*}$ \\
\hline Infectious & $6(20.7)$ & $9(19.1)$ & $0.87^{\star}$ \\
\hline Gastrointestinal & $0(0)$ & $1(2.1)$ & $0.429^{\star}$ \\
\hline Other & $0(0)$ & $1(2.1)$ & $0.429^{\star}$ \\
\hline No symptoms & $0(0)$ & $3(6.4)$ & $0.165^{\star}$ \\
\hline \multicolumn{4}{|l|}{ Hospital stay } \\
\hline Overall LOS \pm SD & $5.31 \pm 5.53$ & $2.15 \pm 2.27$ & $0.001 \dagger$ \\
\hline Intensive care unit admittance, $n$ (\%) & $28(96.6)$ & $3(6.4)$ & $0.000^{*}$ \\
\hline
\end{tabular}

LOS = length of stay in days, represented as mean \pm SD.

${ }^{*}$ Chi square test.

†t-test.

coagulopathy did not improve, and she died after becoming subconscious post-dialysis on day 14 . She did not receive ASV. It was not clear if ASV was considered for this patient. In 2015, no lethal cases were described.

Length of hospital stay. In 2013 , the mean LOS $( \pm S D)$ was $4.01 \pm 5.4$ days compared with $3.36 \pm 4.1$ days in 2015 . This difference was not significant (Table $4, P=0.409$ ). The LOS regarding the type of snakebite also did not differ significantly between those 2 years. In 2015, in comparison with 2013, significantly more patients were admitted in the ICU $(P<$ $0.001)$. In 2015 , the mean LOS $( \pm S D)$ for patients who did not receive ASV $(n=47)$ compared with patients who received ASV $(n=29)$ was $2.15 \pm 2.27$ and $5.31 \pm 5.53$ days, respectively (Table 3). This difference was statistically significant $(P=$ $0.001)$. The mean LOS $( \pm S D)$ for patients who did not receive ASV in $2013(n=67)$ and $2015(n=47)$ was $4.06 \pm 5.44$ and 2.15 \pm 2.27 days, respectively. This difference was statistically significant $(P=0.025)$.

Late complications. Late complications were seen in $18(26.5 \%)$ and $24(31.6 \%)$ patients in 2013 and 2015, respectively (see Table 5), with a significant larger decrease in renal function in 2015. In 2015, 6 (42.9\%) of 14 patients who received ASV on admission developed local complications (e.g., cellulitis, abscess formation, blistering, and necrosis). Patients who developed AKI or a decrease in renal function in 2015 had all received ASV on admission because of VICC. Four of these patients who developed AKI or a decrease in renal function received ASV within 24 hours.

\section{DISCUSSION}

This is the first study from Suriname that reports on the clinical outcomes after ASV administration in an ICU setting. After the introduction of our snakebite protocol with administration of ASV in selected patients with signs of snakebite envenoming, there was no difference in the overall length of hospital stay for 2013 and 2015. However, ASV was given more frequently in 2015 than in 2013 after the introduction of the national snakebite protocol. The frequency of ICU admittance due to snake envenomation increased after the

TABLE 4

Characteristics of snakebite patient hospitalizations for 2013 and 2015

\begin{tabular}{lcr}
\hline & $2013(n=68)$ & $2015(n=76)$ \\
\hline Overall LOS & $4.01 \pm 5.42$ & $3.36 \pm 4.12$ \\
ICU admittance & $1(1.5 \%)$ & $31(40.8 \%)$ \\
ICU LOS & 2 & $0.409 \dagger$ \\
LOS regarding type of snakebite & & $1.90 \pm 2.81$ \\
$\quad$ Identified poisonous snakebite & $3.47 \pm 4.49$ & $3.70 \pm 3.73$ \\
Unidentified snakebite & $4.45 \pm 6.08$ & $3.03 \pm 4.48$ \\
Bothrops atrox & $3.83 \pm 5.02$ & $3.73 \pm 3.84$ \\
Lachesis muta & $2.00 \pm 1.55$ & $0.815 \dagger$ \\
\hline
\end{tabular}

ICU = intensive care unit; LOS = length of stay in days, represented as mean \pm SD

${ }^{*}$ Chi square test.

†t-test. 
TABLE 5

Overview of complications in snakebite victims, including victims who did not receive anti-snake venom, in 2013 and 2015

\begin{tabular}{lccc}
\hline \multicolumn{1}{c}{ Late complications } & $2013, n(\%)$ & $2015, n(\%)$ & \\
\hline Total & $18(26.5)$ & $24(31.6)$ & $0.501^{*}$ \\
Local complications & $14(20.6)$ & $12(15.8)$ & $0.455^{*}$ \\
Decrease in renal function & $1(5.9)$ & $5(6.6)$ & $0.126^{*}$ \\
Late venom-induced consumption & $2(2.9)$ & $6(7.9)$ & $0.195^{*}$ \\
$\quad$ coagulopathy & $1(1.5)$ & $0(0)$ & $0.289^{*}$ \\
latrogenic & $0(0)$ & $1(1.3)$ & $0.343^{*}$ \\
Missing & &
\end{tabular}

introduction of the national snakebite protocol, as a result of the increased administration of ASV and according to the protocol that dictates standardized ICU admittance. The mean length of hospital stay in 2015 for patients who did not receive ASV was shorter than that for patients who did receive ASV. Patients receiving ASV had significantly more symptoms of envenoming, particularly hematologic and metabolic complications were more frequently seen. The length of hospital stay for patients who did not receive ASV in 2015 was significantly shorter than the length of hospital stay in 2013 for patients who did not receive ASV. This may indicate that a clearer distinction in ASV administration in 2015 between patients resulted in a significantly shorter length of hospital stay for those who did not receive ASV. These findings emphasize the benefits of introducing a national snakebite protocol consisting of a standardized and structured evaluation and proper medical treatment of snakebite victims.

In a recent retrospective study in French Guiana, the introduction of a different polyvalent antivenom against venoms from the genera Bothrops, Lachesis, and Crotalus did not show any efficacy of the antivenom on biological and clinical parameters. ${ }^{10}$ An international symposium debating about venomous snakebites in the region was also held in French Guiana in 2016 addressing these issues. Because of the high snakebite mortality rate, they have argued for a reevaluation of their snakebite management protocols and the use of ASV.

There are several preclinical and in vitro studies showing that ASV made from the venom of Latin American vipers neutralizes the effects of the venom, including its procoagulant effect. ${ }^{11,12}$ The efficacy of the ASV in these preclinical studies does not always translate to clinical effectiveness in field settings. The potential reasons for this failure may include antivenom inefficacy, irreversible venom-mediated effects, inability of the antivenom to reach the venom target, rapid venom onset, and mismatch of venom and antivenom pharmacokinetics. ${ }^{13}$

Venom-induced consumption coagulopathy was the most important indication for ASV treatment in our study. Venominduced consumption coagulopathy seemed more prominent in 2015 than 2013, but this difference was only significant for INR $\geq 1.6$. The effect of ASV on the recovery of VICC may vary, partly depending on the type of snake species. ${ }^{4,5,14}$ In African viper envenoming, efficient antivenom administration corrects clotting functions within a few hours. ${ }^{8}$ It takes 12-18 hours for the liver to restore normal levels of clotting factors, even after the venom is neutralized by ASV in the case of VICC. ${ }^{4}$

In 2013, patients with significant VICC were treated with an average of three FFP packages per patient after which normalization of coagulation studies occurred. In 2015, more patients were identified with VICC. These patients received
FFPs with or without ASV to restore coagulation, not leading to a difference in outcome with regard to LOS in the hospital, indicating a possible preventive effect of ASV or that both treatments were at least equally effective. Several studies have indicated that replacement of coagulation factors by administration of FFP speeds up recovery of VICC when administered after administration of ASV. ${ }^{15-17}$ The administration of FFP is not without risk in snakebite envenoming; with the administration of FFP, clotting pathways remained activated despite the administration of antivenom. Also, anaphylaxis, transfusion-related acute lung injury, and risk of blood-borne infections have been described after FFP infusion. ${ }^{4,5}$ Currently, it is advised to only administer FFP in patients with clinical signs of bleeding. ${ }^{5}$ There are no randomized controlled trials studying the efficacy of ASV in the treatment of VICC in comparison to a placebo or observation without intervention. ${ }^{18}$ Anti-snake venom is still the recommended choice of treatment in $\mathrm{VICC},{ }^{4}$ and it can be beneficial for other potential lethal signs of snakebite envenoming such as AKI and neurological complications. ${ }^{1}$

In our study, five patients in 2015 still developed renal dysfunction after the administration of one dosage of ASV due to VICC. Four of these patients received ASV within 24 hours of admission. A delay in the administration of ASV has been shown to increase the risk of developing AKI. ${ }^{19}$ Most patients in both 2013 and 2015 presented within a reasonable time frame of 6 hours after the bite to the emergency department. Although most patients received ASV within 24 hours of admission, it was not possible to identify more specific time lapses between snakebite and ASV administration.

No conclusion could be drawn regarding mortality as there was only one mortality case in 2013 and no fatalities in 2015. One patient in 2013 with a significant patient delay of 48 hours and severe multi-organ failure died due to complications of snakebite envenoming. This patient was not given ASV, although it was not clear if it was considered. Anti-snake venom administration was not a standard procedure at that time. As more patients were given ASV in 2015, we could postulate that if this patient had been treated in a setting with protocolled ASV administration, the patient might have received ASV in an earlier stage of envenomation. Data on out-of-hospital mortality/morbidity and mortality after hospital discharge are not available in Suriname. Also, an unknown number of patients will seek traditional medicine without consulting regular primary health caretakers, before admission or even after discharge from the hospital. In any event, adverse reactions during ASV administration in our patients were mild and did not result in the cessation of ASV administration.

Limitations. Interpretation of our retrospective study results is limited because of a number of reasons. First, 
documentation of patient treatment records was restricted on delay in the administration of ASV and recovery time of VICC and local injury, which is essential to determine the efficacy of ASV. Second, just classification of patients using the traditional snakebite severity score to evaluate the outcome was not possible because of the absence of information on the extent of erythema. Third, documentation on the continuation of treatment of snakebite complications in the outpatient setting was scarce. Fourth, snake species were often unknown, and in cases where it was determined, this was based on patient or bystander recognition, which might be subject to recall bias. Fifth, retrospective grading of envenomation was not possible, and therefore, dry bites could not be excluded in the analyses. Sixth, the absence of patients' follow-up after the administration of ASV is also a crucial limitation. Finally, the lack of the efficacy of the chosen ASV in Suriname could be related to an insufficient dose and/or low potency because there had not been any preclinical studies performed to test the neutralizing ability of this ASV against specific venoms from the snake species that occur in Suriname.

Conclusion and future perspectives. With the protocolled evaluation of snakebite victims, more patients received the protocolled administration of ASV and were admitted to the ICU, contributing to an improved standard of care in Suriname. The protocolled evaluation of snakebite victims also resulted in a shorter length of hospital stay for the patients who did not receive ASV. The administration of ASV in selected patients with signs of snakebite envenoming did not influence the overall length of hospital stay in Suriname's largest hospital responsible for the acute care of snakebite victims, despite more coagulation disorders in the treated group than the historical group. Adverse reactions during ASV administration were mostly mild. There is an urgent need for randomized controlled prospective studies in low- and middle-income countries with proper registration of parameters to evaluate the efficacy of ASV.

Received February 24, 2020. Accepted for publication June 5, 2020.

Published online July 13, 2020.

Acknowledgments: We thank Marja van Eer, internal medicine at the Diakonessenhuis in Paramaribo, Suriname, for her careful and critical review of the manuscript. We also thank Anisma Gokoel, at the Research Center of the Academic Hospital Paramaribo, for her help with the statistical analysis of the data.

Authors' addresses: Navin Ramdhani and Simone Jonker, Department of Intensive Care Unit, Academic Hospital Paramaribo, Paramaribo, Suriname, E-mails: nramdhani@azp.sr and simonejjonker@gmail.com. Kevin van 't Kruys, Academic Pediatric Center Suriname, Academic Hospital Paramaribo, Paramaribo, Suriname, E-mail: kevhenry@ live.com. Rakesh Bansie, Department of Internal Medicine, Academic Hospital Paramaribo, Paramaribo, Suriname, E-mail: rakeshbansie@ yahoo.com. Wilco Zijlmans, Scientific Research Center Suriname, Academic Hospital Paramaribo, Paramaribo, Suriname, and Faculty of Medical Sciences, Anton de Kom University of Suriname, Paramaribo, Suriname, E-mail: wilco.zijlmans@uvs.edu.

\section{REFERENCES}

1. Gutiérrez JM, Calvete JJ, Habib AG, Harrison RA, Williams DJ, Warrell DA, 2017. Snakebite envenoming. Nat Rev Dis Prim 3: 17063.

2. Pinho FMO, Yu L, Burdmann EA, 2008. Snakebite-induced acute kidney injury in Latin America. Semin Nephrol 28: 354-362.

3. Warrell DA, 2010. Snake bite. Lancet 375: 77-88.

4. Maduwage K, Isbister GK, 2014. Current treatment for venominduced consumption coagulopathy resulting from snakebite. PLoS Negl Trop Dis 8: e3220.

5. Berling I, Isbister GK, 2015. Hematologic effects and complications of snake envenoming. Transfus Med Rev 29: 82-89.

6. Mutricy R, Egmann G, Marty C, Houcke S, Adenis A, Douine M, Nacher M, Epelboin L, 2018. Predictors of complications of snake envenomation in Cayenne, French Guiana, 2007-2015. Intensive Care Med 44: 115-117.

7. Mutricy R et al., 2018. High mortality due to snakebites in French Guiana: time has come to re-evaluate medical management protocols. PLoS Negl Trop Dis 12: e0006482.

8. Mion G, Larréché S, Benois A, Petitjeans F, Puidupin M, 2013. Hemostasis dynamics during coagulopathy resulting from Echis envenomation. Toxicon 76: 103-109.

9. Williams DJ, Habib AG, Warrell DA, 2018. Clinical studies of the effectiveness and safety of antivenoms. Toxicon 150: 1-10.

10. Heckmann X, 2017. Place de l' immunothérapie dans les envenimations par crotalinae dans l' ouest guyanais. France: Bulletin de la Société de Pathologie Exotique. To cite this version: HAL Id: dumas-01560643 PAR CROTALINAE DANS L' OUEST GUYANAIS. 0-129.

11. Laines J, Segura A, Villalta M, Herrera M, Vargas M, Alvarez G, Gutiérrez JM, León G, 2014. Toxicity of Bothrops sp. snake venoms from Ecuador and preclinical assessment of the neutralizing efficacy of a polyspecific antivenom from Costa Rica. Toxicon 88: 34-37.

12. Segura $A$ et al., 2010. Preclinical assessment of the neutralizing capacity of antivenoms produced in six Latin American countries against medically-relevant Bothrops snake venoms. Toxicon 56: 980-989.

13. Isbister GK, 2010. Antivenom efficacy or effectiveness: the Australian experience. Toxicology 268: 148-154.

14. Isbister GK, Duffull SB, Brown SGA, 2009. Failure of antivenom to improve recovery in Australian snakebite coagulopathy. QJM 102: 563-568.

15. Brown SGA, Caruso N, Borland ML, McCoubrie DL, Celenza A, Isbister GK, 2009. Clotting factor replacement and recovery from snake venom-induced consumptive coagulopathy. Intensive Care Med 35: 1532-1538.

16. Isbister GK, Buckley NA, Page CB, Scorgie FE, Lincz LF, Seldon M, Brown SGA, 2013. A randomized controlled trial of fresh frozen plasma for treating venom-induced consumption coagulopathy in cases of Australian snakebite (ASP-18). J Thromb Haemost 11: 1310-1318.

17. Holla SK, Rao HA, Shenoy D, Boloor A, Boyanagari M, 2018. The role of fresh frozen plasma in reducing the volume of anti-snake venom in snakebite envenomation. Trop Doct 48: 89-93.

18. Maduwage K, Buckley NA, de Silva HJ, Lalloo DG, Isbister G, 2015. Snake antivenom for snake venom induced consumption coagulopathy. Cochrane Database Syst Rev 2015: CD011428.

19. Singh R, Uraiya D, Kumar A, Tripathi N, 2016. Early demographic and clinical predictors of developing acute kidney injury in snake bite patients: a retrospective controlled study from an Indian tertiary care hospital in North Eastern Uttar Pradesh India. Indian J Crit Care Med 20: 404-408. 